\title{
Employing MALDI-MS on Poly(alkylthiophenes): Analysis of Molecular Weights, Molecular Weight Distributions, End-Group Structures, and End-Group Modifications
}

\author{
J insong Liu, Robert S. Loewe, and Richard D. McCullough* \\ Department of Chemistry, Carnegie Mellon University, Pittsburgh, Pennsylvania 15213 \\ Received April 8, 1999; Revised Manuscript Received J uly 6, 1999
}

\begin{abstract}
Regioregular, head-to-tail coupled, poly(3-alkylthiophenes), synthesized by three different methods, were subjected to MALDI-TOF MS analysis. Polymer samples of both high and low polydispersities were examined. Polymer samples of narrow polydispersity were obtained by fractionation of the polymer by Soxhlet extraction with various solvents. Comparisons between the molecular weights calculated by MALDI and GPC of all fractionated polymer samples showed that GPC calculated molecular weights are a factor of 1.2-2.3 times higher than MALDI. The polydispersities calculated by MALDI were identical or slightly lower than those calculated by GPC. Polymer end-group compositions were also analyzed. We found that more than one type of end-group structure could be detected, and these structures are dependent on the synthetic method employed. Chemical modification of the end-group structure was also performed and monitored by MALDI with successful results. We observed that smaller polymer chains were subject to end-group fragmentation when the MALDI laser power was high. Larger polymer chains, however, showed no such fragmentation.
\end{abstract}

\section{Introduction}

Conjugated polymers have received intense interest due to their practical and potential applications for electronic and photonic materials and devices. Poly(3alkylthiophenes) (P3ATs) represent a large portion of this field because of their good solubility, environmental stability, and processability. Control over the regiochemistry and cross-coupling of 3-substituted thiophene monomers has been achieved by using either organomagnesium ${ }^{1}$ or organozinc ${ }^{2}$ cross-coupling methods on active thiophene intermediates using $\mathrm{Ni}$ (II) catalysts. Such defect-free, structurally homogeneous, head-to-tail coupled polythiophenes (HT-PTs) have improved electronic and photonic properties over regiorandom analogues. ${ }^{1}$ Other synthetic methods have recently appeared that generate HT-PATs using Stille ${ }^{3 a, b}$ or Suzuki ${ }^{3 c}$ cross-coupling methods. Most recently, our lab has developed another synthetic method to generate HTPATs by employing Grignard metathesis (GRI M method Scheme 1) to generate the active cross-coupling intermediate. ${ }^{3 d}$

Although poly(3-alkylthiophenes) can now be considered as well-studied and easily prepared polymers, less attention has been given to the nature of the end groups of such polymers. Information on the end-group composition of such polymers can aid in understanding the termination mechanisms of the polymerization. More importantly, monitoring end-group composition may lead to subsequent chemical modification of these groups which could lead to the preparation of functionalized and/or telechelic poly(3-alkylthiophenes).

Another important issue that needs to be closely examined for PAT systems is their molecular weight (MW) and molecular weight distributions (MWD). ${ }^{4,5}$ Molecular weight parameters of poly(alkylthiophenes) may effect their solid-state morphologies, optical properties, and other physical properties. Determination of the molecular weights of poly(alkylthiophene) systems are al most invariably found by gel permeation chromatography (GPC) using polystyrene standards. GPC is
Scheme 1. A New Synthetic Method Using Grignard Metathesis To Prepare HT-PATs

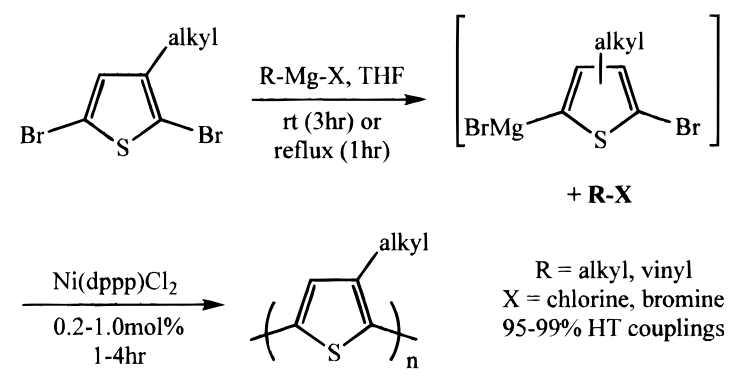

based on correlating the hydrodynamic volume of the randomly coiled polymer chains with polymer molecular weight. Conjugated polymer systems such as polythiophenes, however, adopt a more rodl ike conformation in solution. Because of this, GPC tends to overestimate the molecular weights of such systems. ${ }^{5}$

For these two reasons, we have examined the MALDITOF spectra of poly(alkylthiophenes). MALDI-TOF MS is a recently introduced soft ionization technique that has proven to be a powerful tool for analysis of many biopolymers, such as proteins, oligonucleotides, and polysaccharides.6,7 More recently, it has also been used to characterize many synthetic polymers. ${ }^{8-12}$ It has been shown to be a viable tool for end-group analysis as well as product confirmation for synthetic polymers. Another advantage of MALDI-TOF MS for synthetic polymer analysis is that the absolute molecular masses can be determined as opposed to relative molecular weights by GPC, and this determination is independent of polymer structures. Thus, MALDI-TOF MS appears to be an ideal method for the analysis of rigid-rod synthetic polymers. However, few papers have been found concerning MALDI analysis of conjugated polymer systems. ${ }^{13,14}$ This paper reports on the MALDI analysis of regioregular poly(3-alkylthiophenes) and will demonstrate that MALDI is a powerful tool for end-group monitoring and modification of these systems. 


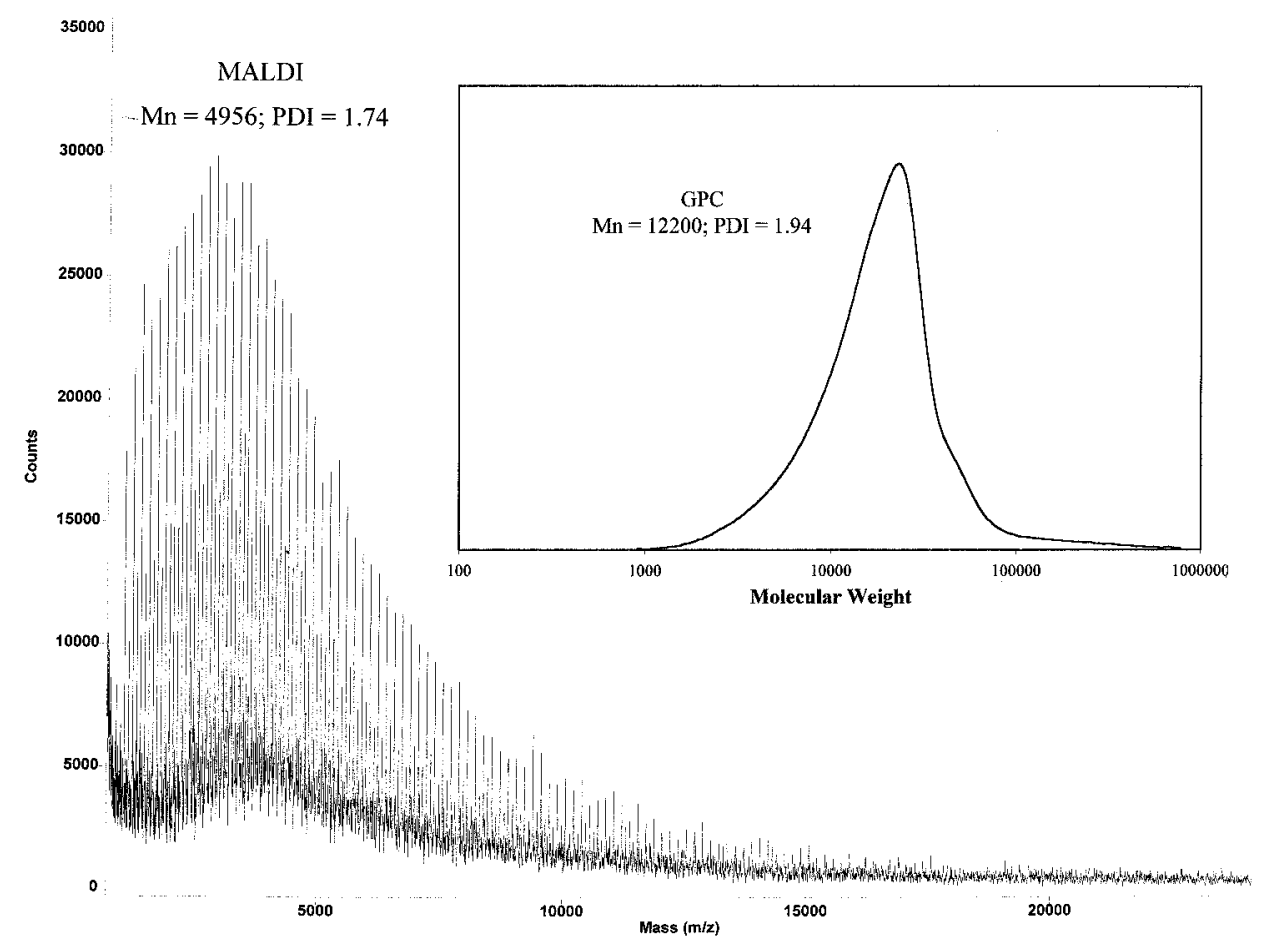

Figure 1. MALDI MS and GPC of an unfractionated sample of P3HT made using ref 1.

\section{Results and Discussion}

Choice of Matrix. Dihydroxybenzoic acid (DHB), dithranol, trans-3-indole acrylic acid (IAA), sinapinic acid, and 9-nitroanthracene were examined as matrices with and without cationization salts. Only dithranol and 9-nitroanthracene were found to assist in the desorption of poly(alkylthiophene) systems. The use of dithranol gave the best results in the analysis of these polymers. Hence, dithranol was chosen as the matrix for all of our polymer samples. ${ }^{15}$

Analysis of MW and MWD. Direct measurement of both the molecular weight (MW) and molecular weight distribution (MWD) of poly(alkylthiophenes) is one of our objectives. Poly(alkylthiophenes) prepared by step growth polymerizations ${ }^{1-3}$ without solvent extraction usually have large polydispersities. Fortunately, the polydispersity can be reduced by subjecting the polymer samples to Soxhlet extractions using a variety of organic solvents. Typically, acetone and hexane are capable of dissolving oligomers and low molecular weight poly(3alkylthiophenes); methylene chl oride can dissolve moderate molecular weight polymers while THF and chloroform can dissolve larger polymer chains. Figure 1 shows the GPC and MALDI spectra of a highly disperse, unfractionated poly(3-hexylthiophene) (P3HT) sample prepared using the McCullough method. ${ }^{1}$ The MWD of these two spectra are markedly different. In the GPC trace, the MWD is skewed toward higher molecular weights, while the MALDI MWD is skewed toward lower molecular weights. One factor that accounts for this difference is that the MALDI signal intensity is proportional to the molar fraction of each species, whereas a GPC curve represents the weight fraction distribution of each species. Other factors include the mass dependence of the desorption/ionization process and the mass-dependent detection efficiency in MALDI . Because of this, higher mass components tend to be underrepresented with respect to lower mass components. ${ }^{16}$ Such mass discrimination is a general problem in the MALDI analysis of highly disperse polymer

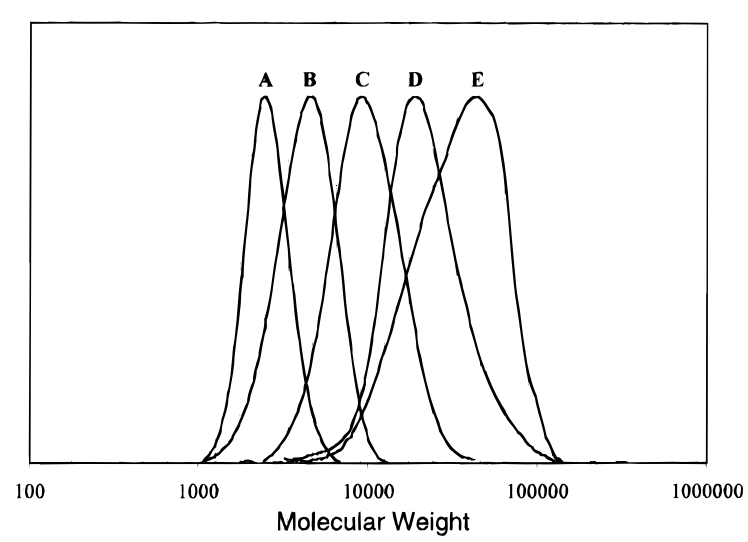

Figure 2. GPC for fractionated samples of P $3 \mathrm{HT}$ (fractionated from $\mathrm{F}$ igure 1 sample): (A) acetone fraction $\left(\mathrm{M}_{\mathrm{n}}=2.4 \mathrm{~K}\right.$, PDI $=1.09)$, (B) hexanes fraction $\left(M_{n}=3.9 \mathrm{~K}, \mathrm{PDI}=1.17\right)$, $(\mathrm{C})$ methylene chloride fraction $\left(\mathrm{M}_{\mathrm{n}}=8.5 \mathrm{~K}, \mathrm{PDI}=1.23\right)$, (D) THF fraction $\left(M_{n}=18.0 K, P D I=1.33\right)$, and $(E)$ chloroform fraction $\left(\mathrm{M}_{\mathrm{n}}=26.5 \mathrm{~K}, \mathrm{PDI}=1.42\right)$.

systems.9,16 Successful analysis of molecular weights and molecular weight distributions for highly disperse polymer samples can be achieved by coupling the GPC data with the MALDI data. ${ }^{17}$ Fractionation of the polymer samples by GPC and subsequent analysis of these fractions by MALDI allows for the $M_{n}$ and $M_{w}$ to be determined for each fraction. Once the average molecular weight of all the fractions is determined by MALDI, subsequent calibration of the GPC curve against the MALDI data allows for the calculation of the molecular weight of highly disperse (unfractionated) polymer samples.

To reduce the polydispersity of our polythiophenes, we simply used the step solvent extraction method reported by Pron, ${ }^{4}$ which fractionated the poly(alkylthiophene) into five samples of narrow polydispersity. After the polymerization, the polymers were precipitated in methanol, filtered, and washed with hot methanol using a Soxhlet apparatus. The polymer was then 


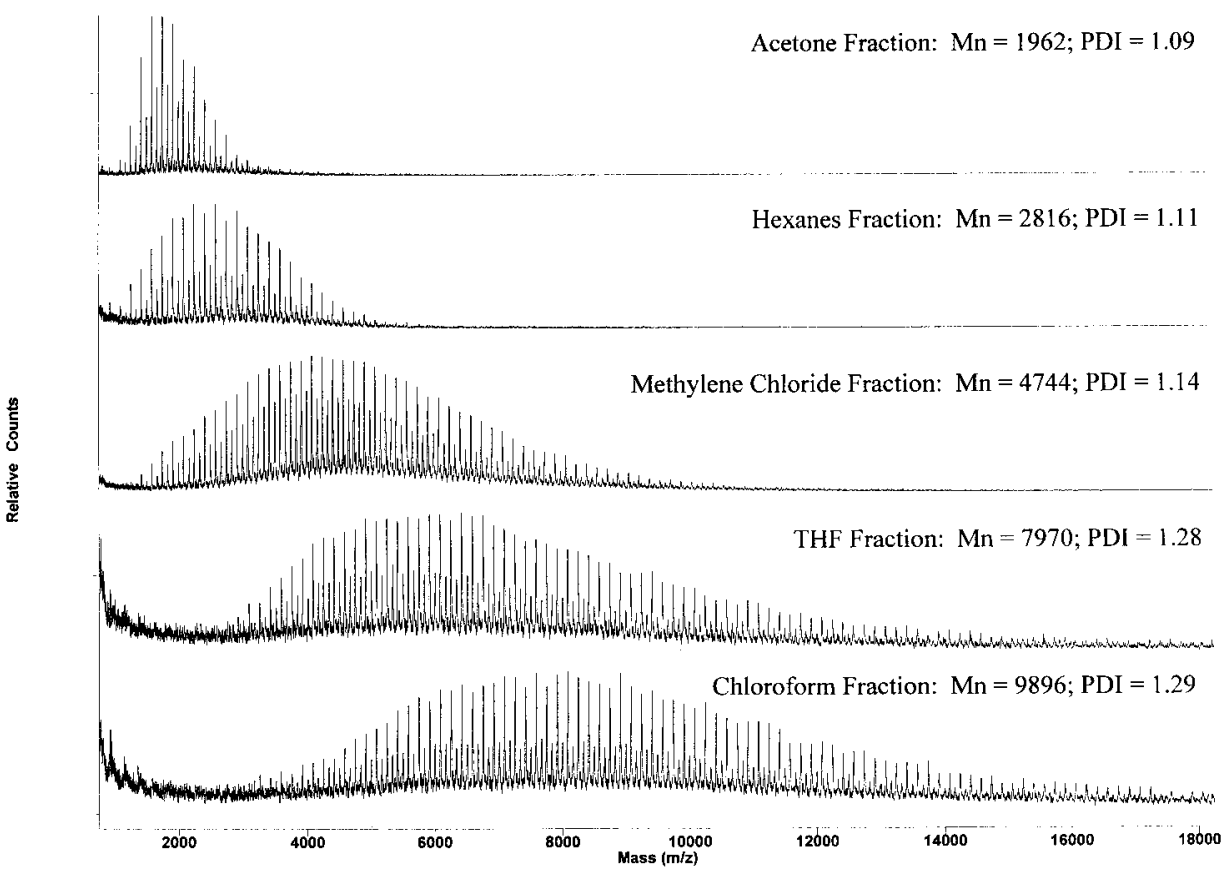

Figure 3. MALDI MS for fractionated samples of P3DDT (fractionated from Figure 1 sample).

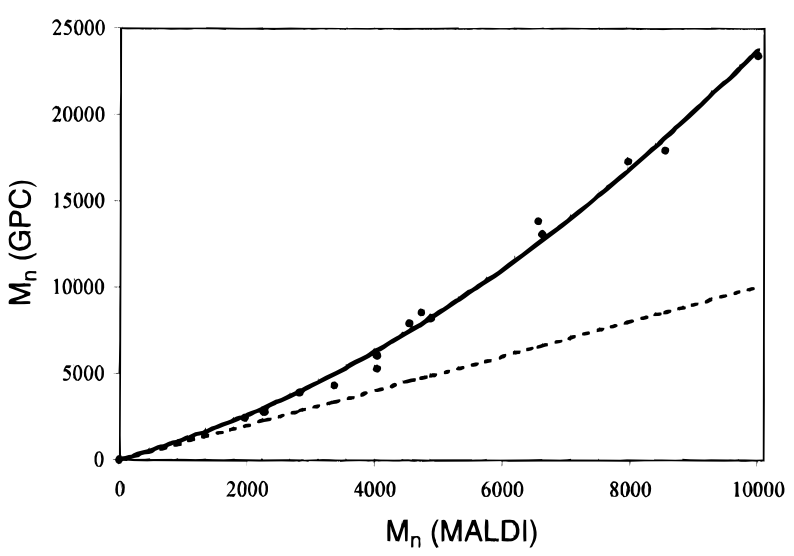

Figure 4. Graph showing the relationship of the molecular weight as calculated by MALDI and GPC. The line serves as a reference (slope $=1$ ).

Soxhlet extracted with acetone, hexane, methylene chloride, THF, and chloroform to provide five fractions. Each fraction was concentrated by rotoevaporation of some of the solvent. The resultant concentrated solutions were cooled to $0{ }^{\circ} \mathrm{C}$ to reprecipitate the polymer.

Figure 2 shows the GPC of each of the five fractions of the P3HT sample from Figure 1. Figure 3 displays the MALDI MS of each of these fractions. Comparison of Figure 2 and Figure 3 shows that a similar MWD can be seen for all fractions. The main differencelies in the absolute molecular weight that each method calculates. For all polymer samples examined, the GPC always gave higher molecular weights than MALDI, presumably due to the shortcomings of GPC for rigidrod polymer systems. The polydispersity index calculated from the MALDI spectra were the same or slightly higher than that of GPC.

Comparisons between the molecular weights of poly(3-hexylthiophenes) calculated by GPC and MALDI were performed for many polymer samples of narrow dispersity. Figure 4 plots the $M_{n}$ as determined by GPC versus the $M_{n}$ as determined by MALDI for such polymers. Comparison of the two $M_{n}$ values using MALDI and GPC shows that the latter always over- estimates the $M_{n}$ and $M_{w}$. When the molecular weight of the polymer is low (acetone and hexane fractions), the $\mathrm{M}_{\mathrm{n}}$ calculated by GPC is 1.2-1.5 times larger than the MALDI results. For higher MW polymer samples, the $M_{n}$ calculated by GPC is 1.5-2.3 times larger than the MALDI results. This result is similar to that seen in the analysis of rigid-rod oligothiophene-ethynylene systems in which the GPC data overestimate the actual molecular weight by a factor of 1.5-2.0. ${ }^{18}$ Comparison of GPC results of poly(3-hexylthiophenes) with embulliometry shows that the GPC overestimates the absolute molecular weight by a factor a 1.8-2.0,5 which is in good agreement with our data.

For higher molecular weight PATs, the MALDI signal intensity is very weak and allows for only a qualitative analysis of the molecular weight. Figure 5 shows the MALDI spectra for a P3HT sample whose $M_{n}$ was calculated to be $75 \mathrm{~K}$ by GPC $\left(\mathrm{M}_{\mathrm{w}}=150 \mathrm{~K}, \mathrm{PDI}=2\right)$. A small peak can be seen between $\mathrm{m} / \mathrm{z}$ of $30000-50000$. The $M_{n}$ of this sample was calculated to be 36K. The molecular weight difference between the MALDI and GPC of this very high molecular weight polymer is 2.1, which falls in the range as shown in Figure 4 . We believe that for very high molecular weight polythiophenes the desorption/ionization efficiency is low (presumably due to the increased degree of aggregation of the polymer) which can help explain the low signal intensities for these samples.

End-Group Analysis. MALDI is also a very powerful tool for end-group analysis and structure confirmation for PAT systems provided that repeat unit resolution can be achieved. In Figures 1 and 3, two major types of signal peaks per repeat unit could be found. The difference between each type of end group was calculated to be $166 \mathrm{Da}$, which confirms that the analyte is poly(3-hexylthiophene) (repeat unit mass $=166.282$ $\mathrm{g} / \mathrm{mol}$ ). Detailed analysis reveals that the masses of each peak can be accurately expressed by $(166 n+1+1)$ and $(166 n+80+1)$ where $n$ is equal to the number of repeat units. From the above information, we can conclude that this polymer sample has only two major types of endgroup configurations. The peak which corresponds to 


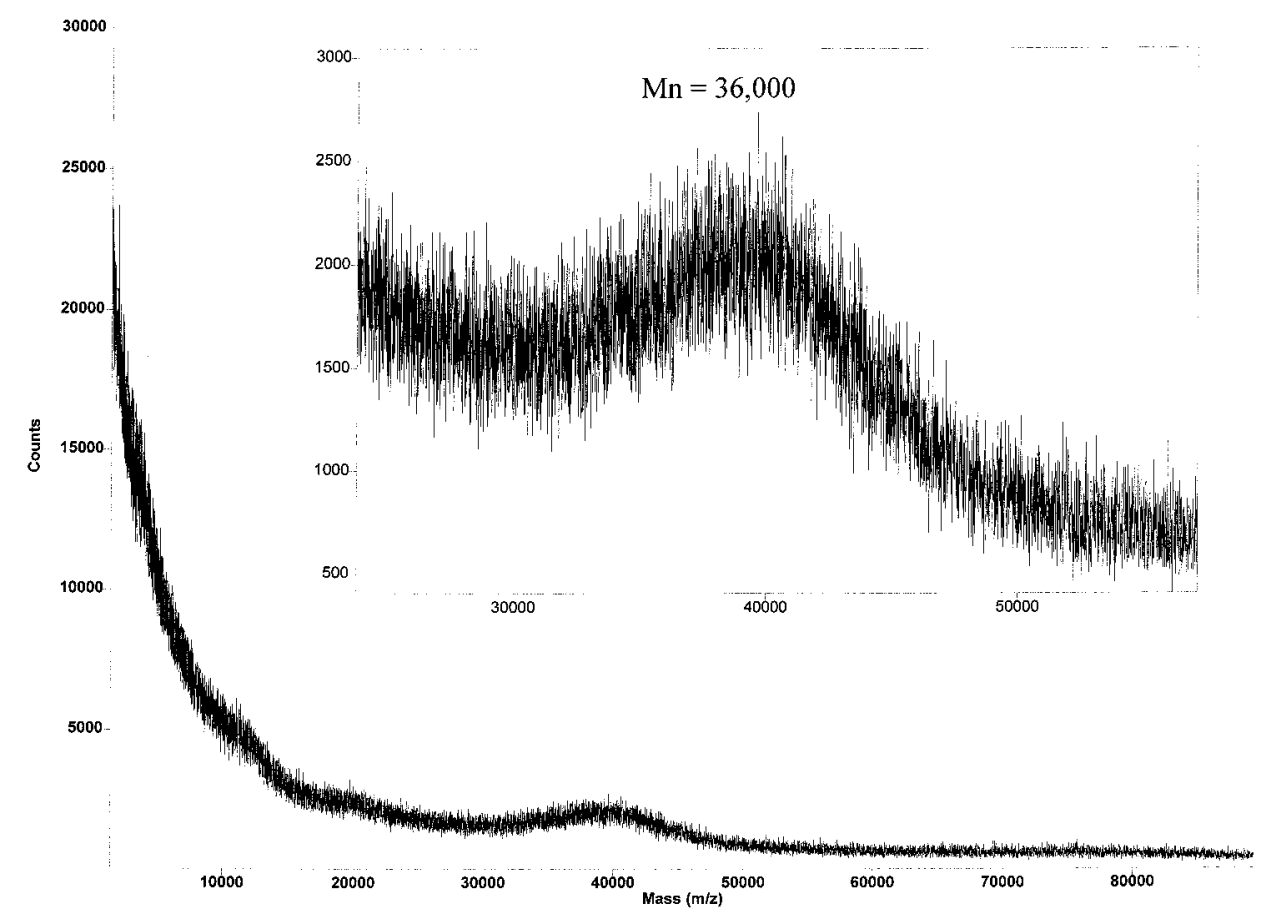

Figure 5. MALDI MS of a very high molecular weight sample of $\mathrm{P} 3 \mathrm{HT}$ made using ref 1.

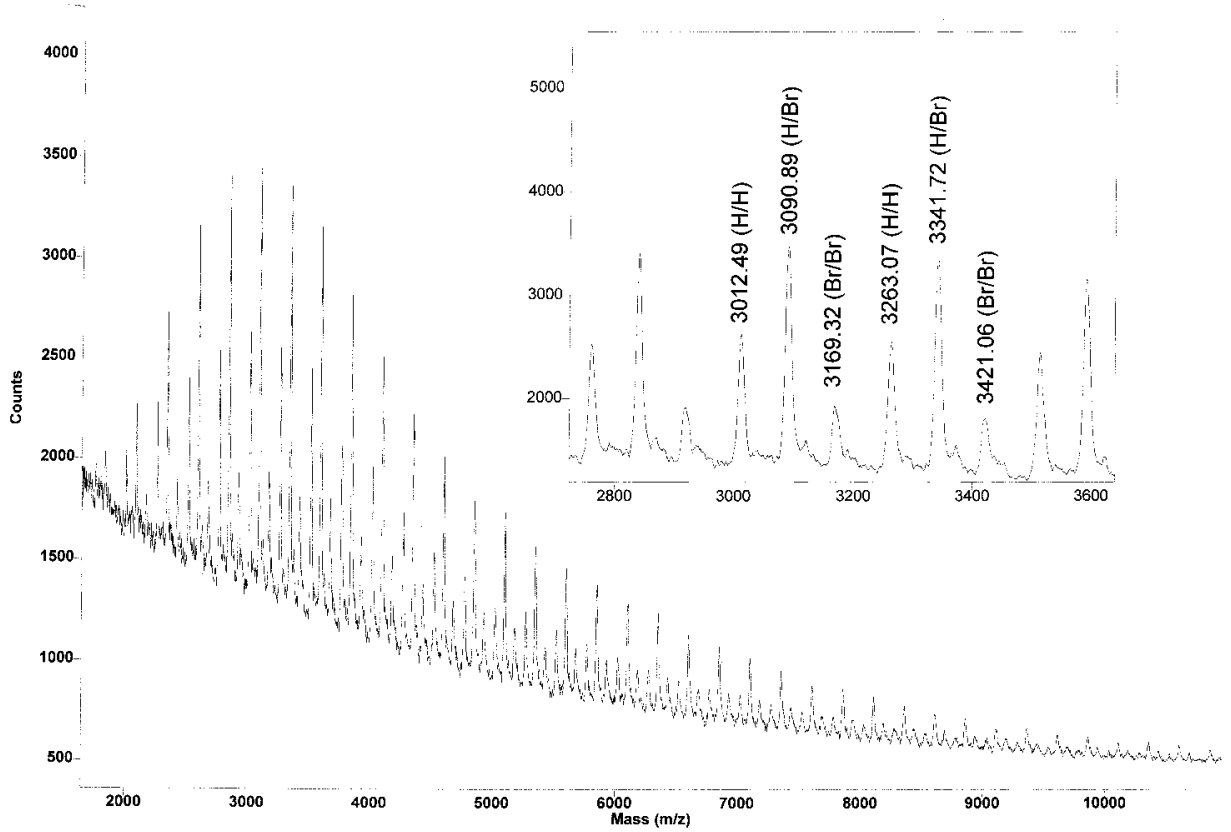

Figure 6. MALDI MS of a sample of P3DDT (THF fraction, made from ref 1 ) shows three types of end-group structures.

$(166 n+1+1)$ corresponds to polymer chains that are terminated by hydrogen atoms at both the 2- and the 5-positions, herein referred to as $\mathrm{H} / \mathrm{H}$. It follows that the peak corresponding to $(166 n+80+1)$ refers to polymer chains terminated by a bromine atom on one end and a hydrogen atom on the other end (referred to as $\mathrm{H} / \mathrm{Br}$ ).

In some polymer samples, a third end-group peak type could be seen which could be expressed by ((repeat unit mass) $n+80+80$ ) which correlates to polymers with bromines terminating both end positions (referred to as $\mathrm{Br} / \mathrm{Br}$ ). For example, Figure 6 shows a MALDI MS for a sample of regioregular poly(3-dodecylthiophene) (HTP3DDT), as prepared by the McCullough method. ${ }^{1}$ it can be seen from this figure that there are three types of end-group structures per repeat unit. Detailed ex- amination shows that they are of type $\mathrm{H} / \mathrm{H}, \mathrm{H} / \mathrm{Br}$, and $\mathrm{Br} / \mathrm{Br}$. We found that the relative abundances of these types of end-group peaks were variable and were largely a function of the polymerization conditions. Specifically, slight changes in the quantity of reagents employed, the monomer purity, the amount of catalyst addition, and polymerization time all affect the distribution of the endgroup structures. For some poly(hexylthiophene) samples we could also observe end groups terminated by bromine atoms at both chain ends, but unfortunately, the resolution between this peak and the $\mathrm{H} / \mathrm{H}$ end-group peak of the next highest repeat unit was poor (mass of a hexylthiophene repeat unit $=166$, mass of two bromine atoms $=160$ ). However, such peaks could be seen as a shoulder in the $\mathrm{H} / \mathrm{H}$ peaks. As a result, only qualitative assignments for this peak could be made. 
Scheme 2. H/Br End Groups Arise from Quenching the Polymerization Reaction with Methanol

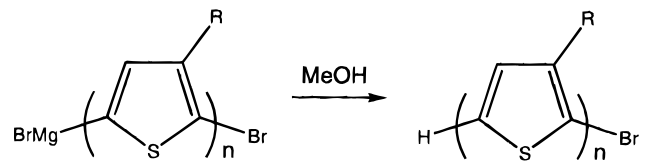

Scheme 3. $\mathrm{H} / \mathrm{H}$ and $\mathrm{Br} / \mathrm{Br}$ End Groups Can Arise from Magnesium-Halogen Exchange $(m>n)$

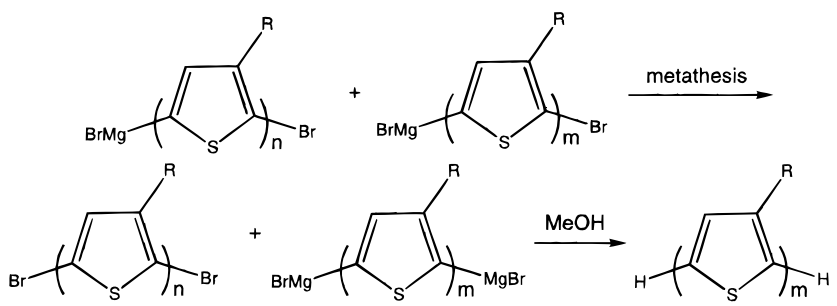

Scheme 4. Br/Br End Groups May Also Arise from Homocoupling
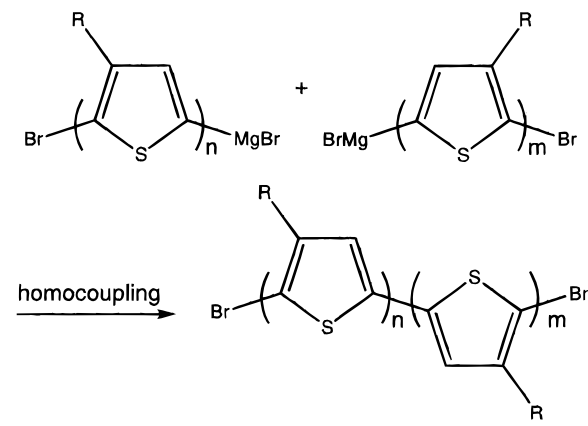

This end-group information can help us understand chain termination mechanisms in Grignard crosscoupling polymerizations. The major type of end group in Figures 3 and 6 is $\mathrm{H} / \mathrm{Br}$. This configuration is likely due to the protonation of the terminal Grignard reagent upon quenching the polymerization reaction with methanol (Scheme 2). Contribution to the $\mathrm{H} / \mathrm{H}$ and $\mathrm{Br} / \mathrm{Br}$ end groups may be a result of halogen-metal exchange between two Grignard species on different polymer chains (Scheme 3). Bromine-magnesium exchange is known to occur on thiophene monomers. ${ }^{3 d, 19,20}$ Specifically, the reaction of an alkyl Grignard reagent with an aryl halide results in the formation of the more stable aryl Grignard reagent and the alkyl halide. Halogenmagnesium exchange will occur provided that the newly formed Grignard reagent is more stable. Oligomeric and polymeric Grignard species may also undergo metathesis, provided that the products are of greater stability. Hence, long polymer chains may undergo metathesis with monomeric or oligomeric species.

The formation of $\mathrm{Br} / \mathrm{Br}$ end groups may also be a result of homocoupling between Grignard reagents on different polymer chains (Scheme 4). Monomer purity has also been found to affect the end-group distribution. Specifically, when 2-bromo-3-hexylthiophene monomers had trace quantities of 3-hexylthiophene (0.2-2\%), the resulting MALDI spectra had a much higher degree of $\mathrm{H} / \mathrm{H}$ end groups than with analytically pure monomers. Last, mass imbalance in the reaction will also affect endgroup distributions. For example, polymerizations that employed an excess of LDA (>1.0 equiv) were found to contain higher $\mathrm{H} / \mathrm{H}$ signal intensities in the MALDI spectra.

We also analyzed poly(alkylthiophene) samples prepared by other methods. Figure 7 shows the MALDI MS
Scheme 5. $\mathrm{H} / \mathrm{CH}_{3}$ End Groups May Arise from Reaction with Unreacted Grignard Reagent

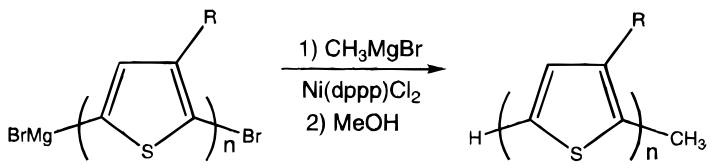

Scheme 6. Synthetic Methods for Removal of End-Group Bromine Atoms in PATs

a)<smiles>[2H]c1cc(C(C)(Br)Br)sc1C(=O)OO</smiles><smiles>[2H]c1cc(C(C)C)sc1C(C)(C)C</smiles>

b)

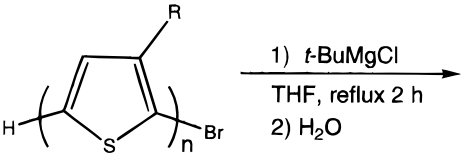<smiles>Cc1cc(C)c(P)s1</smiles>

of the methylene chloride fraction of P3HT (presumably prepared using the polymerization method devel oped by Rieke), which was purchased from Aldrich. Fractionation of this polymer sample was necessary to obtain samples of narrow polydispersity. The GPC of the purchased material showed an $\mathrm{M}_{\mathrm{n}}$ of $35 \mathrm{~K}$ and a PDI of 2.15. This figure shows that there are two types of endgroup signals, which after calculation revealed that they are of type $\mathrm{H} / \mathrm{H}$ and $\mathrm{H} / \mathrm{Br}$ with the $\mathrm{H} / \mathrm{H}$ end-group signal intensity being the strongest. $\mathrm{The} \mathrm{H} / \mathrm{Br}$ end-group signal can be attributed to quenching of the organozinc re agents with methanol or water. Because of the reluctancy of zinc to undergo halogen-metal exchange reactions, we believe that the $\mathrm{H} / \mathrm{H}$ end groups may result from sample impurities such as 2-bromo-3hexylthiophene.

Figure 8 shows the MALDI MS of the hexanes fraction of $\mathrm{P} 3 \mathrm{HT}$, which was polymerized using the Grignard metathesis method (GRIM) recently developed in the McCullough lab ${ }^{3 d}$ (Scheme 1). F or this particular sample, the Grignard reagent used to promote exchange was methylmagnesium bromide. Two major types of end-group peaks can be seen. The mass difference between each type of end group is $166 \mathrm{Da}$, which confirms that the repeat unit is 3-hexylthiophene. The mass difference between adjacent peaks was found to be $65 \mathrm{Da}$. From these data, we believe that the two types of end groups are $\mathrm{H} / \mathrm{CH}_{3}(166 n+1+15)$ and $\mathrm{H} / \mathrm{Br}$ $(166 n+1+80)$ (Figure 8). The $\mathrm{H} / \mathrm{Br}$ end-group signal probably arises due to quenching with methanol. Although the other end-group signal, $\mathrm{H} / \mathrm{CH}_{3}$, is a little more difficult to explain, it is possible that the methyl group can be introduced by coupling of terminal Grignard reagents on the polymer with some unreacted methylmagnesium bromide (Scheme 5). More details on the nature of this polymerization reaction will appear elsewhere. ${ }^{21}$

End-Group Modification. Since the MALDI MS can reveal end-group information on polymer systems, it may also be a useful tool for monitoring end-group modification of poly(alkylthi ophenes). One simple demonstration to illustrate this point is the hydrodebromination of regioregular head-to-tail poly(hexylthiophene) end groups. Scheme 4 shows two methods we have tried as a means to hydrodebrominate the end groups bearing a bromine atom of poly(3-hexylthiophene). In our first attempt (Scheme 6a), poly(3-hexylthiophene) was treated 


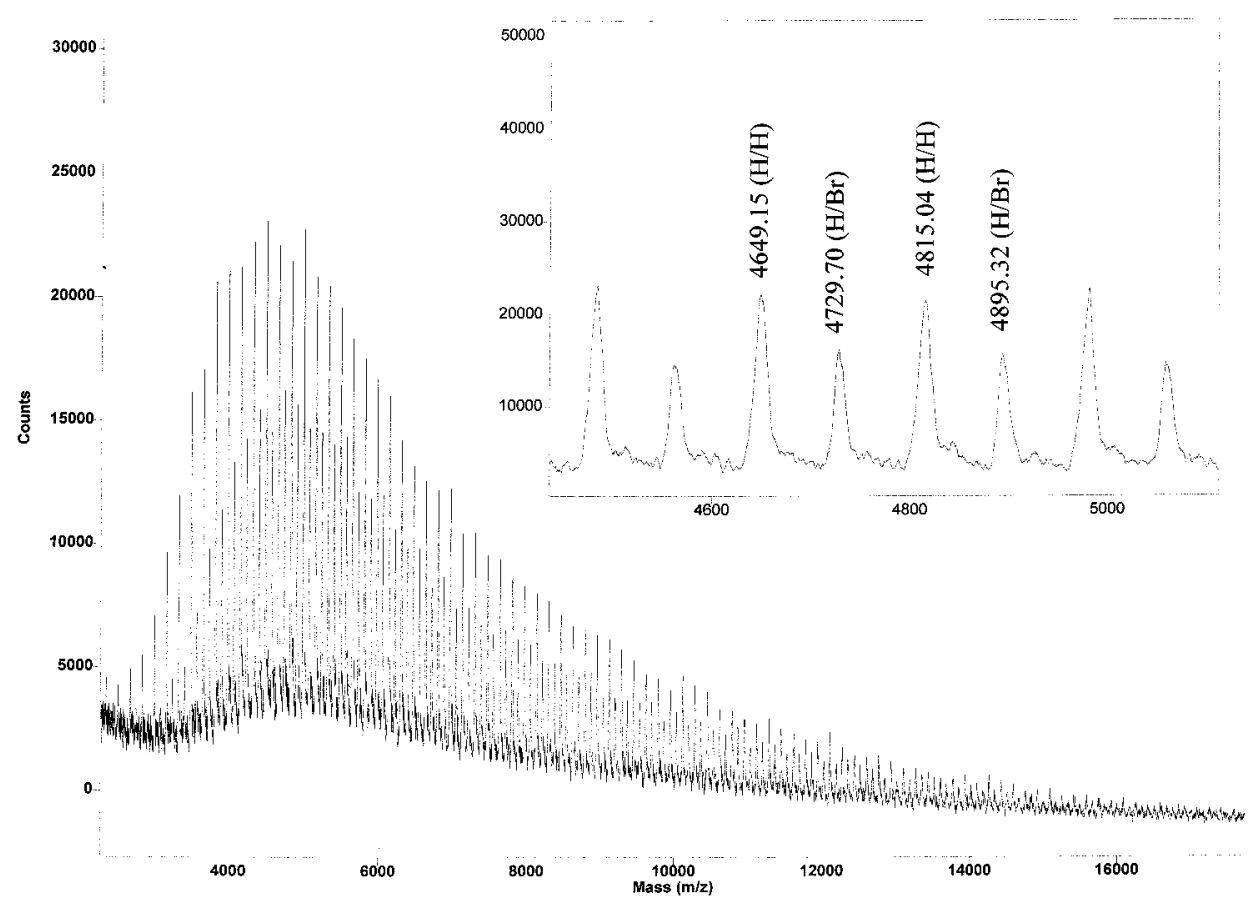

Figure 7. MALDI MS of a sample of $\mathrm{P} 3 \mathrm{HT}$ (methylene chloride fraction) made using Rieke zinc.

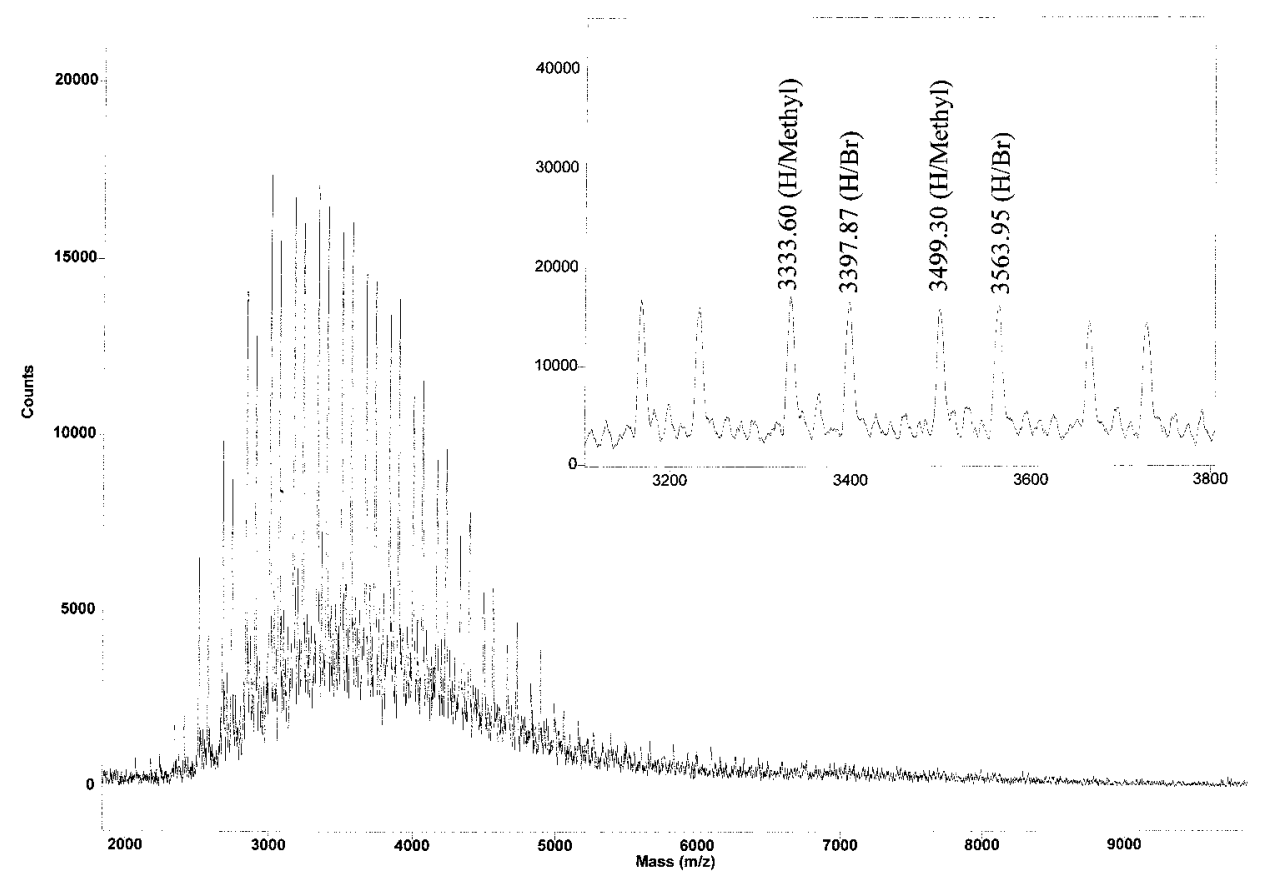

Figure 8. MALDI MS of a sample of P3HT (hexanes fraction) made from Scheme 1.

with zinc dust and acetic acid. After the reaction, the polymer was precipitated from methanol and subjected to MALDI analysis. Figure 9 shows this result. It can be seen that the signal intensity of the $\mathrm{H} / \mathrm{Br}$ peaks has disappeared for the low molecular weight polymer chains. However, for peaks of higher molecular weight, the peaks arising from $\mathrm{H} / \mathrm{Br}$ end groups can still be seen. These data suggest that there was a heterogeneity problem which could be caused by the lower solubility or lower reactivity of larger polymer chains. We believe that solubility is the major reason in this result because the THF/ $/ \mathrm{H}_{2} \mathrm{O} / \mathrm{CH}_{3} \mathrm{COOH}$ mixture does not dissolve high molecular weight polymers very well. Another method to debrominate the polythiophene chains was therefore undertaken (Scheme 6b). In this method, poly(3-hexylthiophene) in THF was treated with t-BuMgCl and heated to reflux for $2 \mathrm{~h}$. Quenching the polymer sol ution with water afforded poly(3-hexylthiophene) with no bromine end groups. Figure 10 shows the MALDI spectra of the polymer before and after Grignard treatment. It can be seen that all of the $\mathrm{H} / \mathrm{Br}$ peaks have been removed, and only $\mathrm{H} / \mathrm{H}$ peaks remain. Further support that the debromi nation reaction was successful can also be seen with elemental analysis. For example, elemental analysis of one polymer sample showed a bromine content $1.56 \%$ before treatment with t-BuMgCl . 


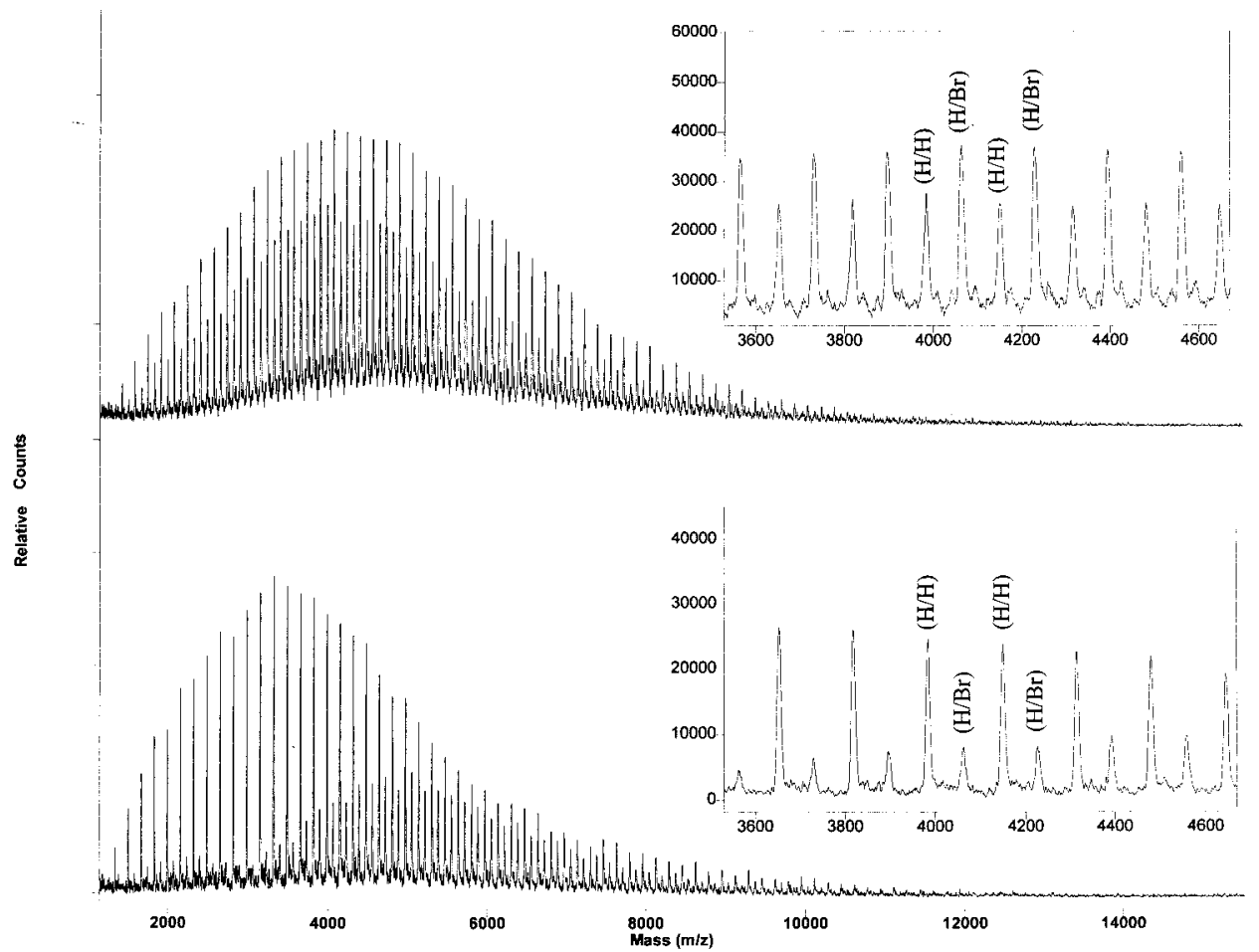

Figure 9. MALDI MS of a sample of P3HT (made by ref 1 ) before (top) and after (bottom) treatment with zinc dust.

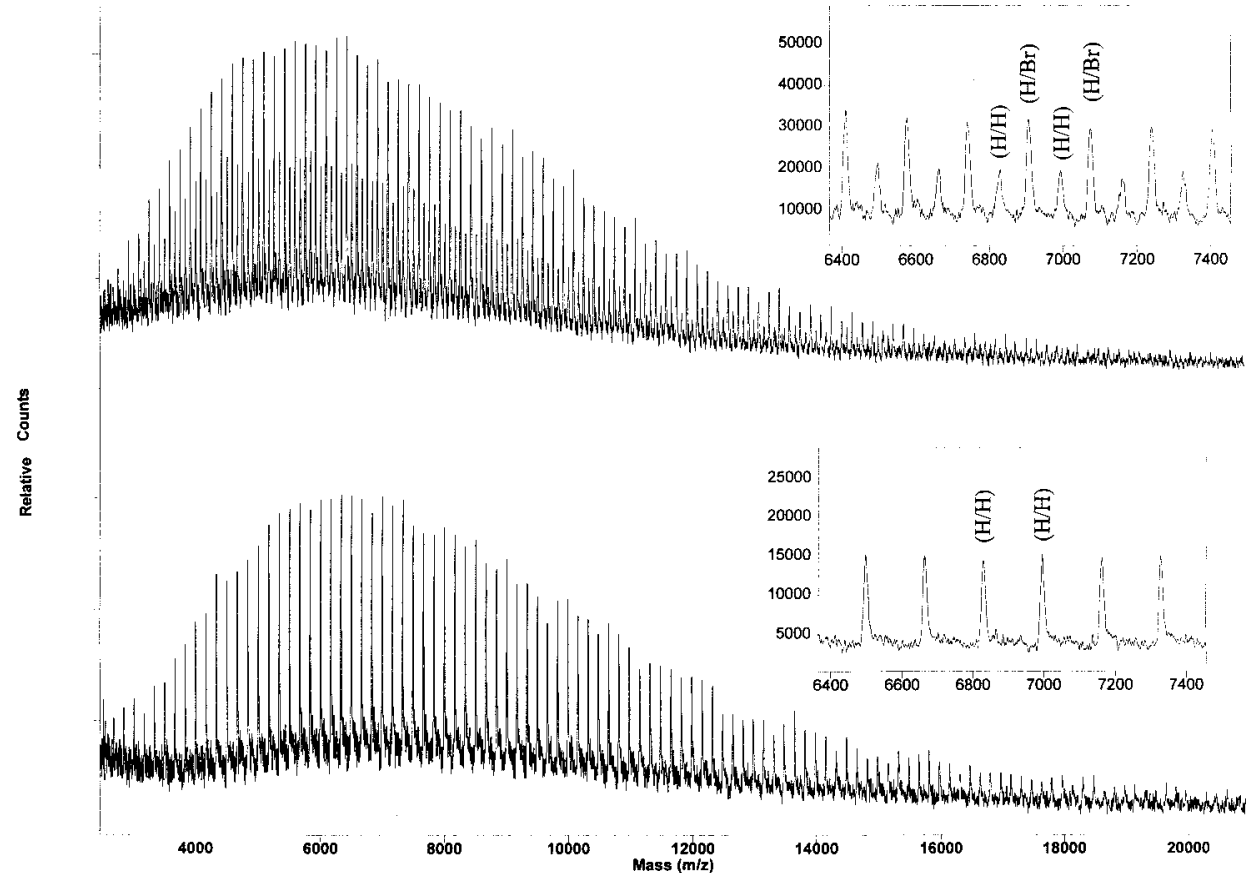

Figure 10. MALDI MS of a sample of P3HT (made by ref 1 ) before (top) and after (bottom) treatment with But $\mathrm{MgCl}$.

Elemental analysis of this polymer after treatment showed no bromine content. These results show that MALDI MS can be used to successfully monitor endgroup modification of poly(alkylthiophene) systems. We are currently performing other end-group modification experiments as well and will report these results el sewhere.

We have also examined many other parameters and studied their subsequent effects on the quality of our MALDI spectra:

Effect of Added Salts. For most MALDI experiments, salt solutions are added to the polymer/matrix solution to aid in the cationization process. ${ }^{22}$ Similar to the observation in MALDI studies on poly-p-phenylene (PPP) systems, ${ }^{13}$ we found that such salts play no effective roles in the cationization of poly(alkylthiophene) systems. In fact, no cationization agent is needed to obtain good MALDI spectra. Three sample preparation methods were examined to see the effects that these salts have (a) using dithranol as matrix without adding any salts, (b) using dithranol, with a sodium chloride solution, and (c) using dithranol, with a silver trifluoroacetate (AgTFA) solution. To each of these solutions was added an appropriate amount of polymer solution. The resultant MALDI spectra yielded almost identical results. For all the spectra, the molec- 
ular cation peaks (166n + end groups) al ways dominated ( $n=$ the number of monomer units). No detection of poly(hexylthiophene) chains carrying a sodium ion $(166 n+$ end group +23$)$ could be found for method $b$. The signals of poly(hexylthiophene) chains carrying a silver ion $(166 n+$ end group +108$)$ could be detected for method c, but their intensity levels are within the noise level. The MALDI spectra recorded using dithranol only was always of better quality than those recorded using dithranol with cationization agents, since the salts usually increased the signal-to-noise ratio.

These results suggest that the predominant cationization mechanism for PATs cannot be attributed to added salt solutions but rather are a result of another ionization process. It has been reported for PPP systems that photoionization plays a major role in the ionization process. ${ }^{13,14}$ We have examined this possibility for our polymer systems and believe that this mechanism does not play a major role in the cationization process. Specifically, PATs have a much lower molar absorptivity at $337 \mathrm{~nm}$ when compared to dithranol. Second, very high matrix:analyte ratios $\left(>10^{5}: 1\right)$ do not diminish the strong polymer signal intensities in the MALDI. Wealso do not observe a high degree of fragmentation like those seen with PPP systems. Although we believe that photoionization is not the major mechanism for ionization, the cationization mechanism for poly(alkylthiophene) systems remains unclear to us. However, it seems possible that the gas-phase collisions between matrix molecules and polymer chains may result in charge transfer, causing the polymer chains to become oxidized to radical cations (polarons). This could explain why the molecular cation signals were so intense and no obvious destructive fragments were observed in our MALDI spectra. Evidence for el ectron-transfer ionization in electroactive molecules has recently been shown by $\mathrm{McC}$ arley et al. ${ }^{23} \mathrm{M}$ ore investigations are needed in order to fully address the exact ionization mechanism in the MALDI process of PATs.

Effect of Laser Power. We also wanted to explore the possibility of end-group fragmentation during the desorption/ionization process. Such a process, if present, could alter the distribution of end-group signal intensity creating inaccuracies in the end-group determinations. To test whether end-group fragmentation was present, we examined peak area ratios as a function of the laser power of the MALDI. We found that, in some cases of low MW polymer samples, there was a direct correlation between the $\mathrm{H} / \mathrm{H}: \mathrm{H} / \mathrm{Br}$ peak ratio as a function of the laser power. Specifically, the ratio between the $\mathrm{H} / \mathrm{H}$ peak to $\mathrm{H} / \mathrm{Br}$ peak increased with increasing laser power, suggesting that the end-group bromine atoms may undergo fragmentation during the desorption/ ionization process and give some contribution to the $\mathrm{H} / \mathrm{H}$ signal.

However, it was not known whether the fragmentation process produced bromine anions or bromine radicals. To see whether the fragmentation process produced bromine anions, we examined the negative ion mode MALDI MS to see whether any bromide ions were present. No such peak could be detected, suggesting that bromine end-group fragmentation for these polymers is a radical process. If this is true, then the new end-group structure would be $\mathrm{H} / \mathrm{radical}$, corresponding to a hydrogen atom on one end of the polymer chain and a radical at the other end. Such an $\mathrm{H} / \mathrm{radical}$ peak would bear an almost identical mass to that of $\mathrm{H} / \mathrm{H}$, making these two peaks virtually indistinguishable. However, when the polymer had a higher molecular weight (for example, THF and chloroform fractions), the laser power had no obvious effect in the ratio of $\mathrm{H} / \mathrm{H}$ to $\mathrm{H} / \mathrm{Br}$ peak intensity. It is probable that the larger polymer chains are able to disperse the energy over a greater number of bonds, extending their intact lifetime and reducing the chance of fragmentation. ${ }^{9}$ Therefore, laser powers sl ightly above threshold values are recommended in the examination of end-group composition, especially for lower molecular weight PAT systems.

Effect of Matrix/Analyte Ratio. Ratios of matrix to analyte were also investigated to see the effects on the resulting spectra. We had expected that a very high matrix to analyte concentration ratio was needed to ensure dilution and separation of the poly(alkylthiophene) chains, because regioregular PATs have a strong tendency to self-assemble. Our experimental results showed that ratios as low as 100:1 (matrix: analyte) gave successful MALDI results. However, without matrix assistance, no MALDI peaks could be detected. We also examined some low mol ecular weight PAT samples prepared from three different matrix/ analyte ratios (200:1, 2000:1, $20000: 1)$ to see whether the matrix/analyte ratio has an effect on the end-group fragmentation. By keeping the laser power constant, we observed no change in the peak area ratio of $\mathrm{H} / \mathrm{H}$ to $\mathrm{H} / \mathrm{Br}$ for each sample, suggesting that the matrix/ analyte ratio does not play a role in end-group fragmentation. This result may suggest that photoionization is unlikely to play a major role in the cationization process of PAT systems.

\section{Conclusions}

We have demonstrated that poly(3-alkylthiophenes) can be successfully characterized using MALDI-TOF MS. The ionization process is different from "normal" MALDI, in which cationization salts are needed. It is likely that charge transfer is the predominant cationization mechanism. Through step solvent extraction and selective reprecipitation, PAT fractions of narrow molecular weight polydispersities can be obtained. The absolute molecular weights of these fraction samples were cal culated by MALDI. Comparing with the MALDI measurements, GPC usually overestimates the molecular weight of rigid-rod PATs by a factor of 1.2-2.3. MALDI spectra can clearly show the end-group composition of PAT samples, which can aid in the elucidation of the termination mechanisms that are present. The polymerization method and/or polymerization conditions can lead to a large variation in the end-group composition of PATS. MALDI could become a powerful tool for monitoring end-group transformations of PATs. Such end-group engineering could lead to the development of new and novel materials. For low molecular weight polymers, we observe some end-group fragmentation. For such cases, low laser power is recommended for more accurate end-group analysis.

\section{Experimental Section}

Materials. All matrices and salts were purchased from Aldrich Chemical Co. Regioregular poly(3-hexylthiophene) (presumably made using Rieke zinc) was purchased from Aldrich. All other polymer samples were synthesized in our laboratory with the McCullough method and the GRIM method.

Instrumentation. MALDI-TOF MS was performed using a Voyager-DE STR BioSpectrometry Workstation by PerSep- 
tive Biosystems. All spectra were recorded using linear ion mode, in which samples were irradiated under high vacuum using a nitrogen laser (wavelength $337 \mathrm{~nm}, 2$ ns pulse). The accelerating voltage was $25 \mathrm{kV}$. Grid voltage and low mass gate were $85.0 \%$ and $500.0 \mathrm{Da}$, respectively. All spectra were collected from 256 laser pulses and signal averaged to give one spectrum. Average molecular weights $\left(M_{n}, M_{w}\right)$ were determined according to the following equations:

$$
\begin{gathered}
\mathrm{M}_{\mathrm{n}}=\sum\left(\mathrm{N}_{\mathrm{i}} \mathrm{M}_{\mathrm{i}}\right) / \sum \mathrm{N}_{\mathrm{i}} \\
\mathrm{M}_{\mathrm{w}}=\sum\left(\mathrm{N}_{\mathrm{i}} \mathrm{M}_{\mathrm{i}}{ }^{2}\right) / \sum\left(\mathrm{N}_{\mathrm{i}} \mathrm{M}_{\mathrm{i}}\right)
\end{gathered}
$$

where $\mathrm{N}_{\mathrm{i}}$ and $\mathrm{M}_{\mathrm{i}}$ represent the number and the mass for the oligomer containing i monomers, respectively. Polydispersities were calculated from the $\mathrm{M}_{\mathrm{w}} / \mathrm{M}_{\mathrm{n}}$ ratio.

All GPC spectra were recorded using a Waters 6000A apparatus and a Waters R410 differential refractometer. The eluent was THF which was kept at $30^{\circ} \mathrm{C}$. The flow rate was $1.0 \mathrm{~mL} / \mathrm{min}$. The system was calibrated using polystyrene standards obtained from polymer standards service.

Sample Preparation. Polymer samples for MALDI analysis were prepared by combining the matrix and analyte in a 10 000:1 ratio. The cationization salt solution of 0.1 M AgTFA or $\mathrm{NaCl}$ in THF was added to some samples. The poly(alkylthiophene) samples were dissolved in THF to yield concentrations of approximately $10^{-4}-10^{-5} \mathrm{M}$. The concentration of the matrix solution was kept at $0.1 \mathrm{M}$. Mixing equal volumes from the matrix and polymer solutions gave the resultant polymer/matrix solution which was used to spot the MALDI sample plate.

Attempted Hydro-debromination of HT-PHT End Groups Using Zinc Dust. Poly(3-hexylthiophene) (200 mg), prepared using the McCullough method, ${ }^{1}$ was charged into a $100 \mathrm{~mL}$ flask with $45 \mathrm{~mL}$ of THF, $10 \mathrm{~mL}$ of water, and $5 \mathrm{~mL}$ of acetic acid. An excess of zinc dust $(4.0 \mathrm{~g})$ was added, and the solution was heated to reflux and stirred for $6 \mathrm{~h}$ before it was poured over $200 \mathrm{~mL}$ of methanol. After precipitation and filtration, the polymer was washed with methanol several times and then was subjected to MALDI analysis.

Successful Hydro-debromination of HT-PHT End Groups Using t-BuMgCl. Poly(3-hexylthiophene) (800 mg), prepared using the McCull ough method, ${ }^{1}$ was dissolved in 100 $\mathrm{mL}$ of dry THF. To this solution was added an excess $(8 \mathrm{~mL})$ of t-BuMgCl (1.0 M in THF). The solution was then heated to reflux and stirred for $2 \mathrm{~h}$ before it was quenched with water. After neutralization with acetic acid, the solution was poured over $300 \mathrm{~mL}$ of methanol, and the precipitated polymer was washed with methanol several times and subject to MALDI analysis.

Acknowledgment. We are grateful to the NSF (CHE-9509959; CHE-9808188) for financial support. We thank Dr. Mark Bier for technical support. We also thank Lubrizol for providing a research fellowship for R.S.L.

\section{References and Notes}

(1) (a) McCullough, R. D.; Lowe, R. D. J . Chem. Soc., Chem. Commun. 1992, 70. (b) McCullough, R. D.; Lowe, R. D.; J ayaraman, M.; Anderson, D. L. J . Org. Chem. 1993, 58, 904. (c) McCullough, R. D.; Tristram-Nagle, S.; Williams, S. P.; Lowe, R. D.; J ayaraman, M. J . Am. Chem. Soc. 1993, 115, 4910.

(2) (a) Chen, T. A.; Rieke, R. D. J . Am. Chem. Soc. 1992, 114, 10087. (b) Chen, T. A.; O'Brien, R. A.; Rieke, R. D. Macromolecules 1993, 26, 3462. (c) Chen, T. A.; Wu, X.; Rieke, R. D. J . Am. Chem. Soc. 1995, 117, 233.

(3) (a) McCullough, R. D.; Ewbank, P. E.; Loewe, R. S. J . Am Chem. Soc. 1997, 119, 633. (b) Iraqi, A.; Barker, G. W. J Mater. Chem. 1998, 8, 25. (c) Guillerez, S.; Bidan, G. Synth. Met. 1998, 93, 123. (d) Loewe, R. S.; Khersonsky, S. K.; McCullough, R. D. Adv. Mater. 1999, 11 (3), 250.

(4) Trznadel, M.; Pron, A.; Zagorska, M.; Chrzaszcz, R.; Pielichowski, J . Macromolecules 1998, 31, 5051.

(5) Holdcroft, S. J . Polym. Sci., Part B 1991, 29, 1585.

(6) Cotter, R. J. Anal. Chem. 1992, 64, 1027.

(7) Danis, P. O.; Karr, D. E.; Mayer, F.; Holle, A.; Watson, C. H. Org. Mass Spectrom. 1992, 27, 843.

(8) Danis, P. O.; Karr, D. E.; Westmoreland, M. C.; Piton, M. C Christie, D. I.; Clay, P. A.; Kable, S. H.; Gilbert, R. G. Macromolecules 1993, 26, 6684.

(9) Thomson, B.; Suddaby, K.; Rudin, A.; Lajoie, G. Eur. Polym. J. 1996, 32, 239.

(10) Gooden, J. K.; Gross, M. L.; Mueller, A.; Stefanescu, A. D.; Wooley, K. L. J . Am. Chem. Soc. 1998, 120, 10180.

(11) Montaudo, G.; Montaudo, M. S.; Puglisi, C.; Samperi, F. Macromol ecules 1995, 28, 4562.

(12) Burkoth, A. K.; Anseth, K. S. Macromol ecules 1999, 32, 1438

(13) Räder, H. J .; Spickermann, J .; Kreyenschnidt, M.; Müllen, K. Macromol. Chem. Phys. 1996, 197, 3285.

(14) Remmers, M.; Müller, B.; Martin, K.; Räder, H. J . Macromol ecules 1999, $32(4), 1073$.

(15) Most recently, we have employed terthiophene as a matrix for polythiophene systems and found it to be as good as dithranol.

(16) Montaudo, G.; Montaudo, M. S.; Puglisi, C.; Samperi, F. Rapid Commun. Mass Spectrom. 1995, 9, 453.

(17) Montaudo, G.; Garozzo, D.; Montaudo, M. S.; Puglisi, C.; Samperi, F. Macromolecules 1995, 28, 7983.

(18) Pearson, D. L.; Schumm, J . S.; Tour, J . M. Macromolecules 1994, 27, 2348

(19) Gronowitz, S.; Cederlund, B.; Hornfeldt, A.-B. Chem. Scr. 1974, 5, 217.

(20) Boymond, L.; Rottlander, M.; Cahiez, G.; Knochel, P. Angew. Chem., Int. Ed. Engl. 1998, 37, 1701.

(21) Loewe, R. S.; Ewbank, P. C.; Liu, J .; McCullough, R. D., manuscript in preparation.

(22) (a) Bahr, U.; Deppe, A.; Karas, M.; Hillenkamp, F. Anal. Chem. 1992, 64, 2866. (b) Spickermann, I .; Martin, K.; Räder H. J .; Müllen, K. Eur. Mass Spectrom. 1996, 2, 161. (c) Räder, H. J .; Schrepp, W. Acta Polym. 1998, 49, 272.

(23) McCarley, T. D.; McCarley, R. L.; Limbach, P. A. Anal. Chem. 1998, 70 (20), 4376.

MA9905324 